P. Schirmacher

W. E. Fleig

H. P. Dienes, für die Konsensusgruppe

„Bioptische Diagnostik der chronischen Hepatitis“*

\title{
Bioptische Diagnostik der chronischen Hepatitis -
}

Ergebnisse einer evidenzbasierten Konsensuskonferenz der Deutschen Gesellschaft für Pathologie (DGP), der Deutschen Gesellschaft für Verdauungs- und Stoffwechselkrankheiten (DGVS) und des Kompetenznetzes Hepatitis (HepNet)

Biopsy Diagnosis of Chronic Hepatitis - Results of an Evidence-Based Consensus Conference of the German Society of Pathology (DCP), the German Society for Digestive and Metabolic Diseases (DGVS), and the Network of Competence for Hepatitis (HepNet)

\section{Schlïisselwörter}

Hepatitis · Biopsie · Konsensus · Grading · Staging · Leber
Key words

Hepatitis · biopsy $\cdot$ consensus $\cdot$ grading $\cdot$ staging $\cdot$ liver

\section{Einleitung}

Die Leberbiopsie ist eine wesentliche Maßnahme in der diagnostischen Abklärung einer chronischen Hepatitis. Ihre klinische Relevanz kann trotz erheblicher Fortschritte in Biopsietechnik, Pathomorphologie und Molekularpathologie durch Mängel in Technik, semiquantitativer Evaluation (Grading, Staging) und Terminologie eingeschränkt sein. Vor dem Hintergrund steigender und zunehmend differenzierter therapeutischer Optionen ist eine Anpassung an den aktuellen Erkenntnisstand, Standardisierung und Qualitätssicherung erforderlich, um den diagnostischen Nutzen der Leberbiopsie zu optimieren und ihren Einsatz zu rechtfertigen.

Deshalb wurde auf Initiative der Deutschen Gesellschaft für Pathologie (DGP, AG Gastroenterologische Pathologie) in Zusammenarbeit mit dem bundesweiten Kompetenznetz Hepatitis (HepNet) und der Deutschen Gesellschaft für Verdauungs- und Stoffwechselkrankheiten (DGVS) eine Konsensusfindung initiiert, die unter Berücksichtigung der klinischen und pathologischen Anforderungen eine dem derzeitigen Erkenntnisstand entsprechende, standardisierte, bioptische Diagnostik und Terminologie beschreiben sollte. Nach Zusammenstellung der Expertenkommission (unter Einbeziehung von Experten aus Österreich und der deutschsprachigen Schweiz) erfolgten eine Befragung aller Teilnehmer und die Erstellung einer ersten Diskussionsvorlage (Juli-September 2002). Anschließend erfolgte ein offenes Expertenhearing mit anschließender Podiumsdiskussion in Form eines gemeinsamen Workshops von DGVS, DGP (AG Gastroenterologische Pathologie) und HepNet während der Jahrestagung der DGVS (Bonn, 12.9.2002). Die Ergebnisse des Workshops wurden in eine zweite Diskussionsvorlage integriert (Oktober-November 2002). Über diese Vorlage mit ihren Konsensusaussagen wurde in einer Konsensuskonferenz aller beteiligten Experten (Köln, 30.11.2002) diskutiert und abgestimmt. Nach Integration dieser Ergebnisse und Verfassung der Erläuterungstexte (Dezember 2002-Mai 2003) erfolgte die abschließende Vorstellung und Diskussion in den Fachgesellschaften (Juni-September 2003). Zusätzlich wurden im Konsensusprozess folgende Informationen berücksichtigt:

1. wissenschaftliche Publikationen, insbesondere soweit sie im anhängenden Literatur aufgeführt sind;

2. bestehende Konsensusstatements, soweit sie das Themengebiet betreffen. Dies sind im Einzelnen:

*Alle Autoren und Mitglieder der Konsensusgruppe sind am Ende der Publikation aufgeführt. 
a) EASL International Consensus Conference on Hepatitis C (Paris, 26.-28.2.1999) - Consensus Statement, publiziert in [1];

b) Behandlung der chronischen Virushepatitis B/D und der akuten und chronischen Virushepatitis C. Konsensus der Deutschen Gesellschaft für Verdauungs- und Stoffwechselkrankheiten (Halle, November 1996, publiziert in [2] [Neufassung des Konsensus in Bearbeitung]);

c) National Institutes of Health Consensus Development Conference Statement: Management of Hepatitis C: 2002, publiziert in [3], http://consensus.nih.gov/cons/116/ $091202116 c d c \_s t a t e m e n t . h t m l ;$

d) International Autoimmune Hepatitis Group Report: review of criteria for diagnosis of autoimmune hepatitis, publiziert in [4].

In allen Konsensusaussagen (fett unterlegt) wurde im Rahmen des Konsensustreffens unter den beteiligten Experten Einigkeit erzielt. Die Erläuterungen dienen der weiteren Information für nicht spezifisch mit der Materie Vertraute; sie waren nicht Gegenstand eines formalen Konsensus, wurden jedoch unter allen Mitgliedern der Expertenkommission abgestimmt. Der formulierte Konsensus fasst den gegenwärtigen Stand der Erkenntnisse zusammen; eine Überarbeitung wird jedoch zum gegebenen Zeitpunkt erforderlich sein.

\section{Definitionen}

\section{Konsens}

Eine Hepatitis ist eine Entzündung der Leber, die durch eine variable Kombination von rundzelliger, vorwiegend lymphozytärer entzündlicher Infiltration, hepatozellulärer Schädigung sowie Regeneration der Hepatozyten charakterisiert wird. Die morphologischen Kriterien der Chronizität sind eine portale Prädominanz der entzündungszelligen Infiltration und jeweils fakultativ eine portale Faservermehrung und die sog. Grenzzonen-(,Interface“)-Hepatitis (Empfehlungsklasse B).

\section{Erläuterung}

Eine wesentliche Implikation der Definition ist die Tatsache, dass unabhängig von der klinischen und serologischen Konstellation die Diagnose einer Hepatitis (d.h. manifesten Leberentzündung) nur dann zu stellen ist, wenn ein hepatozellulärer Zellschaden oder zumindest eine adäquate entzündungszellige Infiltration nachweisbar sind. Der alleinige Nachweis viraler Nukleinsäuren oder Antigene sichert lediglich die virale Infektion, ist jedoch für die Diagnose einer Hepatitis nicht ausreichend. Andererseits kann histologisch durchaus die Diagnose einer chronischen Hepatitis auch bei normwertigen oder nahezu normwertigen Serumwerten (Aminotransferasen; insbesondere bei chronischer Hepatitis C) gestellt werden. Ferner kann eine Hepatitis nur dann diagnostiziert werden, wenn der hepatozellulärschädigende Charakter der Entzündung zumindest im Vordergrund steht. Entzündungen, die vorwiegend andere Leberstrukturen betreffen (z.B. Gallengänge, Gefäße), sollten auch beim Vorliegen einer geringergradigen hepatozellulärschädigenden Komponente nicht als Hepatitis bezeichnet werden. Zur Frage der Chronizität s.u.

\section{Indikationen}

\section{Konsens}

Die Leberbiopsie ist eine wichtige Maßnahme zur Diagnose und Verlaufsbeurteilung einer chronischen Hepatitis (C). Die Indikation wird im klinischen Kontext gestellt (C). Die bioptische Diagnostik dient der Klärung folgender Fragen (C):

1. Diagnose einer Hepatitis und deren Chronizität

2. Bestimmung der entzündlichen Aktivität (Grading)

3. Bestimmung des Fibroseausmaßes (Staging)

4. Aussagen zur Ätiologie

\section{Erläuterung}

Maßgebliche Grundlage der Biopsieentscheidung ist die Frage, ob das diagnostische Ergebnis der Biopsie voraussichtlich für das kurz- und/oder längerfristige therapeutische Vorgehen von Bedeutung ist (s. auch Neufassung des Konsensus der Deutschen Gesellschaft für Verdauungs- und Stoffwechselkrankheiten zur Behandlung der chronischen Virushepatitis B/D und der akuten und chronischen Virushepatitis C). Bei einer Hepatitis dient die Leberbiopsie in erster Linie der Bestimmung der entzündlichen Aktivität (Grading) und des Fibroseausmaßes (Staging) und ist in der Beurteilung dieser Parameter nach wie vor der „Gold-Standard“ " 5,6$]$. Hilfreich ist die Leberbiopsie auch bei normwertigen Aminotransferasen, da der negative prädiktive Wert einer einmaligen bzw. mehrmaligen Aminotransferasenbestimmung für das Vorliegen einer Fibrose nur 33 bzw. $42 \%$ beträgt [7]. Sie ist serologischen Tests in der Fibroseevaluation überlegen und besitzt prädiktive Bedeutung für das therapeutische Ansprechen $[6,8]$. Bei unklarer Anamnese und fehlenden biochemischen und serologischen Vorbefunden kann die Leberbiopsie auch zur Beurteilung der Chronizität einer Lebererkrankung beitragen. Die Beurteilung der Ätiologie ist insbesondere bei unklaren oder negativen serologischen Parametern vor allem in Bezug auf eine chronische Virushepatitis von Bedeutung. Da alle vier o.g. Parameter Einfuss auf die Prognose und Therapie(entscheidung) bei einer chronischen Hepatitis haben können, ist zu jedem Punkt pathologisch-diagnostisch explizit Stellung zu beziehen.

\section{Entnahme und Bearbeitung des Biopsiegewebes}

\section{Konsens}

Die Aussagekraft der Leberbiopsie wird maßgeblich durch die Menge an gewonnenem Lebergewebe bestimmt (B). Für die histologische Beurteilung ist eine Biopsielänge von mindestens $1,5 \mathrm{~cm}$ bei einem Nadeldurchmesser von 1,2-1,8 mm anzustreben (C). Eine adäquate Fixierung muss sich unmittelbar anschließen. Der sachgerechte Auftrag zur Begutachtung erfordert die Mitteilung der relevanten klinischen und serologischen Befunde (C). Die histologische Gewebsbeurteilung muss auf einer ausreichenden Zahl an Schnittstufen basieren und eine adäquate Darstellung des Faser- und Eisengehalts mittels Spezialfärbungen einschließen (C).

\section{Erläuterung (s. Tab. 1)}

Grading und Staging (s.u.) setzen eine ausreichende Biopsiegröße voraus. Da eine direkte Beziehung zwischen Biopsiegröße und Zahl erfasster Portalfelder besteht [9], sollte eine Zylinderlänge von $15 \mathrm{~mm}$ nicht unterschritten werden. Diese Zylinderlänge bei 
Tab. 1 Technische Voraussetzungen

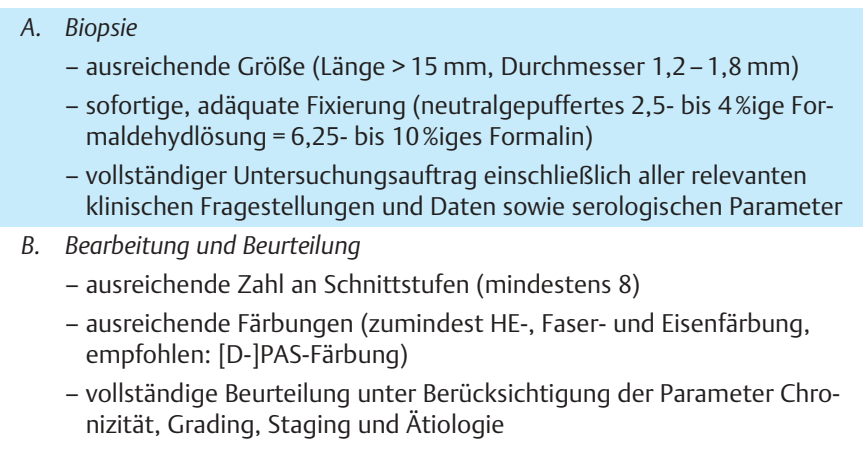

einem Nadeldurchmesser von 1,2 -1,8 mm sichert das Vorhandensein von mehr als 10 beurteilbaren Portalfeldern [9]. Dünnere Nadeldurchmesser $(<1,2 \mathrm{~mm})$ verringern die Aussagekraft. Bei eingeschränkter Gerinnung kann die Biopsie transvenös, z. B. transjugulär erfolgen. Die Verwendung größerer Nadeln (z.B. 2,1 mm, $14 \mathrm{G}$ ) ist mit höheren Komplikationsraten behaftet. Bei Kindern wird ein Nadeldurchmesser von 1,2 mm empfohlen. Während bei der Tru-cut-Biopsie eine Schneidetechnik zur Anwendung kommt, wird durch die Menghini-Nadel ein Lebergewebszylinder angesaugt. In der bislang größten Serie betrugen die Komplikationsraten 3,5:1000 für die Tru-cut-Nadel und 1:1000 für die Menghini-Nadel [10]. Angaben zur Blutungsinzidenz schwanken von 0,06 bis $1,7 \%$, zur Letalität von 0,009 bis $0,33 \%$. Weitere seltene Komplikationen sind Pneumothorax und biliäre Peritonitis. Geringfügige Komplikationen, wie asymptomatische Blutungen oder Postpunktionsschmerz, treten häufiger auf [11, 12]. Bei besonderen Risikofaktoren (hepatische oder systemische Malignome, HIV-Infektion, Niereninsuffizienz, schwere Einschränkung der Leberfunktion, angeborene Blutgerinnungsdefekte) ist die Blutungsrate höher [10,13 - 16]. Eine kürzlich erschienene Arbeit [17] hat zusätzlich mykobakterielle Infektionen, Heparininjektion oder prophylaktische Substitution von Thrombozyten vor Biopsie und die Therapie mit Kortikosteroiden oder Methamizol als unabhängige Risikofaktoren für eine Blutung identifiziert. Außerdem traten Blutungen in 70\% der Fälle verzögert (24 h nach Biopsie oder später) auf. Die Komplikationen können durch eine ausführliche Anamnese und ein standardisiertes Punktionsverfahren minimiert werden [18].

Nach der Biopsieentnahme schließt sich die Fixierung in einer ausreichenden Formalinmenge (Verhältnis Biopsie zu Formalin: mind. $1: 20)$ unmittelbar an. Als Standardfixans wird neutralgepufferte 2,5- bis 4\%ige Formaldehydlösung (entspricht 6,25-10\% Formalin) empfohlen. Abweichungen vom Fixierungsprotokoll sollten nur in Absprache mit dem beurteilenden Pathologen erfolgen.

Mit dem Begutachtungsauftrag sind dem beurteilenden Pathologen die klinische Diagnose und ggf. Differenzialdiagnose sowie folgende dringend erforderliche Parameter mitzuteilen: Labordaten (Transaminasen, $\gamma \mathrm{GT}$, AP, Gammaglobulinkonzentration, Autoantikörper- und Nukleinsäurenachweise) sowie anamnestische Angaben zur Dauer der Erkrankung und zu möglichen zu- sätzlich schädigenden Faktoren (z.B. Alkoholkonsum, Medikamente) sowie alle weiteren Parameter, die aus klinischer Sicht einen Einfluss auf die Ausprägung und die Beurteilung der Hepatitis haben können.

Die histologische Beurteilung sollte auf der Begutachtung von mindestens acht Schnittstufen basieren und neben der Hämatoxylin-Eosin-(HE-)Färbung zumindest eine adäquate Faser(z.B. modifizierte Gomori-Färbung, van-Gieson-Trichromfärbung, Siriusrot-Färbung) und eine Eisenfärbung beinhalten. $\mathrm{Zu}$ sätzlich wird die Anfertigung einer PAS-Färbung (bevorzugt mit Diastasevorbehandlung) empfohlen.

Entitäten

\section{Konsens}

Als Entitäten der chronischen Hepatitis werden ätiologisch abgegrenzt: virale Infektionen (insbesondere HBV/[HDV] und HCV), Arzneimittel und Toxine, Autoimmunität sowie andere, seltene Ursachen (B). Die alkoholische (ASH) und nichtalkoholische (NASH) Steatohepatitis werden nicht im Rahmen des vorliegenden Konsensus erfasst (C).

\section{Erläuterung (s. Tab. 2)}

Der chronischen Hepatitis können verschiedene Ätiologien zugrunde liegen:

a) virale Infektionen: Weltweit führende Ursache der chronischen Hepatitis sind Infektionen mit dem Hepatitis-B-Virus (HBV; evtl. zusammen mit dem Hepatitis-Delta-Virus [HDV]) und dem Hepatitis-C-Virus (HCV). Andere Viren sind als Erreger einer chronischen Hepatitis selten (EpsteinBarr-Virus, fragl. Cytomegievirus);

b) Arzneimittel und Toxine: Mehrere Medikamente können eine chronische Hepatitis als unerwünschte Wirkung verursachen (z. B. Nitrofurantoin, Clometazin, Sulfonamide);

c) Autoimmunität: Autoimmunhepatitis (allein oder [seltener] in Kombination mit zusätzlichen Gallenwegsschädigungen in Form eines sog. Overlap-Syndroms).

d) Stoffwechselerkrankungen: Der M. Wilson manifestiert sich in einem erheblichen Teil der Fälle unter dem Bild einer chronischen Hepatitis. Eine eigenständige Hepatitis infolge eines Alpha-1 Antitrypsinmangels ist fraglich;

e) seltene Ursachen und sog. kryptogene chronische Hepatitis (bei Kindern sollte auch die Möglichkeit einer angeborenen Stoffwechselkrankheit geprüft werden).

Tab. 2 Ätiologie der chronischen Hepatitis

A. chronische Virusinfektion (HBV [evtl. HDV-Koinfektion], HCV, selten: EBV, CMV (?)

B. Medikamente und Toxine (Nitrofurantoin, Clometazin, Sulfonamide u. a.)

C. Autoimmunität (autoimmune Hepatitis, autoimmunes Overlap-Syndrom: AlH-PBC, AlH-PSC [sehr selten])

D. Stoffwechselerkrankungen (M. Wilson, alpha1-Antitrypsin-Mangel [?])

E. seltene Ursachen und "kryptogene" chronische Hepatitis (nonA-E-Viren [?])

konkurrierende Erkrankungen (z. B. Hinweise auf chronischen Alkoholabusus, nichtalkoholische Steatohepatitis, Eisenspeicherkrankheit) beachten! 
Der Nachweis einer HBV- bzw. einer HCV-Infektion reicht nicht zur Diagnose einer chronischen Hepatitis aus (s. o.). Die HBV-Infektion verläuft in bis zu $10 \%$ der Fälle, eine HCV-Infektion in $70-80 \%$ der Fälle chronisch. Sowohl bei der HBV- als auch der HCV-Infektion sind spontane Viruseliminationen dokumentiert. Eine zusätzliche HDV-Infektion kann den Verlauf einer HBV-Infektion verschlechtern.

Auch eine alkoholische (ASH) oder nichtalkoholische Steatohepatitis (NASH) können die Definition einer chronischen Hepatitis in wesentlichen Teilen erfüllen. Beide Entitäten wurden aus folgenden Gründen nicht in die vorliegende Konsensusfindung miteinbezogen:

- klar unterscheidbare Morphologie in Bezug auf entzündungszellige Reaktion (prominent granulozytäre Komponente, evtl. sog. lipophage Granulome), Fibrosemuster (vorwiegend perisinusoidal) und Topographie der Verfettung;

- definierte Unterschiede in Pathogenese, Verlauf, Therapie und Biopsieindikationsstellung;

- fehlende Anwendbarkeit aller aufgeführten diagnostischen Grading- und Staging-Scores bei alleiniger ASH oder NASH.

Dennoch ist besonders auf Kombinationsschädigungen (z. B. ASH oder NASH in Kombination mit Hepatitis C) zu achten, da diese den Verlauf einer chronischen Hepatitis verschlechtern.

\section{Histomorphologische Evaluation - Allgemeines}

\section{Konsens}

Die Beurteilung einer Leberbiopsie bei einer Hepatitis erfordert neben technischen Angaben (Biopsiegröße, Partikelzahl, Zahl der Portalfelder, evtl. Erhaltungszustand) Aussagen zur Frage der Chronizität, zum Ausmaß der entzündlichen Aktivität (Grading), zu Architektur und Fasergehalt (Staging) und zu Ätiologie und Differenzialdiagnose. Sind diagnostische Aussagen eingeschränkt oder unmöglich, so ist dies mit Angabe der Gründe festzuhalten. Vorbiopsien sollten bei der Bewertung berücksichtigt werden (C).

\section{Erläuterung (s. auch Tab. 5)}

Zu den einzelnen Aspekten der histomorphologischen Evaluation wird im weiteren Verlauf Stellung bezogen. Ein Grading und Staging gemäß der u. g. Kriterien ist auch beim Vorliegen kombinierter Schädigungsbilder (z. B. Hepatitis C und ASH) vorzunehmen.

\section{Chronizität}

\section{Konsens}

In der pathomorphologischen Diagnose ist die Frage der Chronizität zu beantworten (C).

\section{Erläuterung}

Die Unterscheidung einer akuten von einer chronischen Hepatitis ist aus ätiologischer, prognostischer und therapeutischer Sicht relevant. Die Diagnose der Chronizität beruht auf zwei nicht deckungsgleichen Sichtweisen: der klinischen und der histopathologischen Definition. Gemäß der klinischen Definition ist eine Hepatitis als chronisch anzusehen, wenn ihre Symptome oder Serumparameter über einen Zeitraum von mindestens 6 Monaten persistieren. Die klinische Definition bietet eine wesentliche Grundlage für die Indikationsstellung zur Biopsie. Ihre Aussagekraft ist aber mit der histopathologischen Chronizitätsdiagnose nicht deckungsgleich (z. B. Unsicherheit von Zeitangaben, mögliche Überlagerung mehrerer akuter Schädigungsbilder (z.B. akute Virushepatitis und akuter medikamentös-toxischer Leberparenchymschaden), Vorliegen definitiver histologischer Chronizitätszeichen vor dem Ablauf von 6 Monaten).

Die histopathologischen Kriterien der Chronizität sind portale Prädominanz der vorwiegend rundzelligen, entzündungszelligen Infiltration, sowie jeweils fakultativ eine portale Faservermehrung und die sog. Grenzzonenhepatitis, d.h. entzündungszellige Infiltration und hepatozelluläre Zelluntergänge in der parenchymatösen Grenzlamelle („Interface-Hepatitis“, früher als sog. Mottenfraßnekrosen bezeichnet).

\section{Ätiologie}

\section{Konsens}

Eine definitive Festlegung der Ätiologie einer chronischen Hepatitis ist oft aufgrund morphologischer Kriterien allein nicht möglich (B); sie ergibt sich aus der Zusammenschau klinischer, serologischer und morphologischer Befunde (B).

\section{Erläuterung (Abb. 1)}

Eine Stellungnahme zur Ätiologie einer chronischen Hepatitis ist bei der pathomorphologischen Befundung obligat. Diese kann definitiv erfolgen, wenn eindeutige Gewebsmerkmale (Milchglashepatozyten oder sog. „,sanded nuclei“ bei chronischer Hepatitis B; immunhistologischer Nachweis viraler Antigene oder molekularpathologischer Nachweis viraler Nukleinsäuren) vorliegen. Es ist jedoch legitim und Ausdruck einer rationellen, interdisziplinären Diagnostik, sich auf verlässlich mitgeteilte, serologische Befunde (als solche zu kennzeichnen) zu beziehen. Auf Diskrepanzen bzw. untypische Veränderungen ist jedoch hinzuweisen. Ein zusätzlicher Nachweis viraler Antigene oder von Nukleinsäuren im Gewebe bei bekannter und eindeutiger Serumkonstellation und kompatiblem histologischem Bild ist nicht notwendig.

\section{Grading}

\section{Konsens}

Der Grad der entzündlichen Aktivität ist definiert durch das Ausmaß des zum Biopsiezeitpunkt bestehenden nekroinflammatorischen Gewebsschadens. Dieser sollte in einem diagnostischen Score festgehalten werden. Akzeptiert sind die Scoringsysteme nach Desmet et al. [19], Batts und Ludwig [20], Ishak et al. (sog. modifizierter HAI-Score) [21] sowie Bedossa et al. (sog. METAVIR-Score; nur für die Hepatitis C) [22]. Es wird empfohlen, den Score nach Desmet et al. [19] anzuwenden. Die Verwendung der früher üblichen Diagnosen „chronisch-persistierende Hepatitis“ und „chronisch-aktive/aggressive Hepatitis“ [23] ist obsolet (C). 

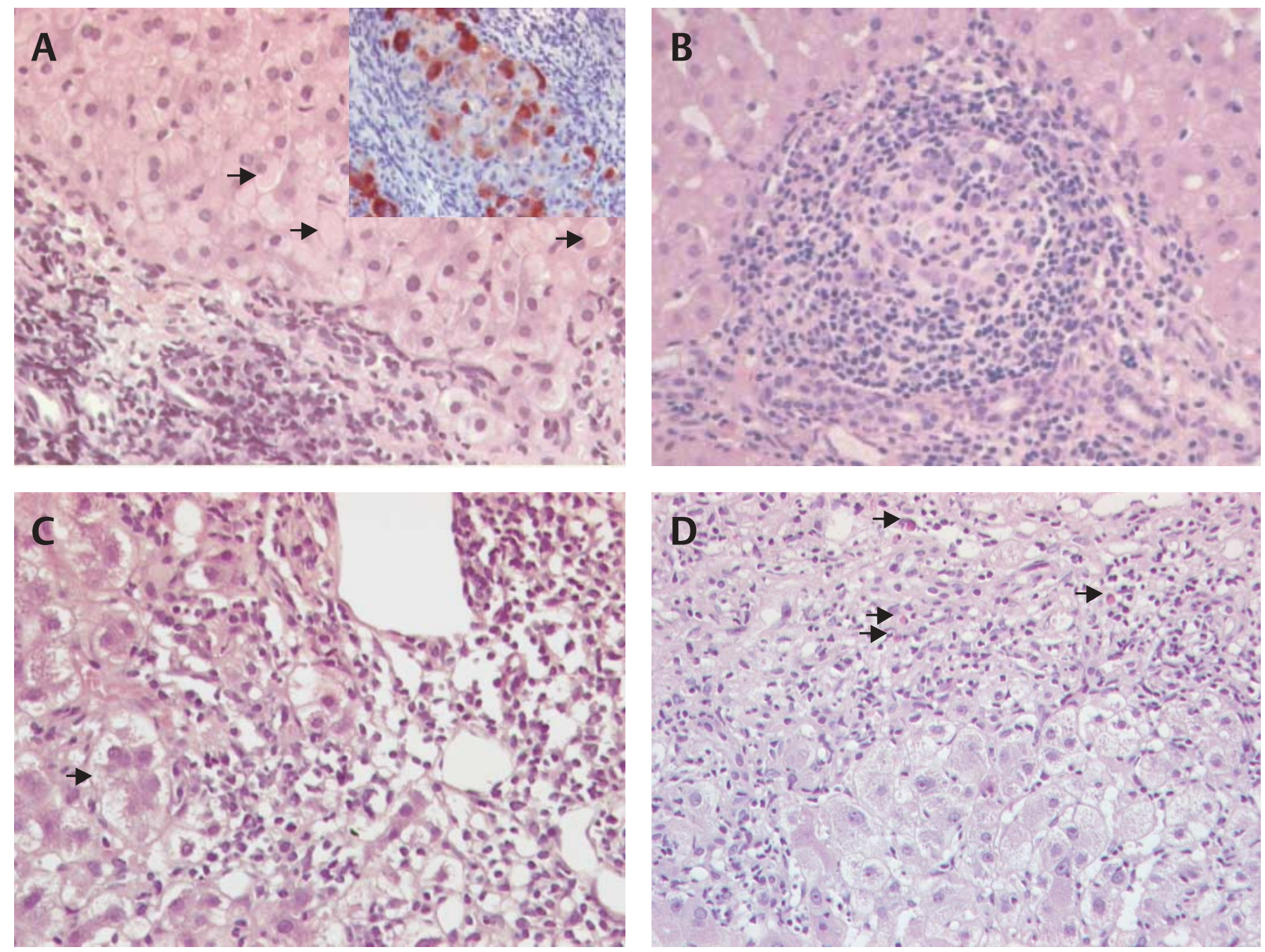

Abb. 1 Ursachen der chronischen Hepatitis (s. auch Tab. 2; HE-Färbung).

(A) Chronische Hepatitis B mit milder entzündlicher Aktivität (Grad 2 nach Desmet) und Nachweis von sog. Milchglashepatozyten (Pfeile); Insert: immunhistologischer Nachweis von HBsAg. (B) Chronische Hepatitis C mit milder entzündlicher Aktivität (Grad 2 nach Desmet); geringe lymphozytäre, portale Entzündungszellinfiltration mit Lymphfollikelbildung bei intakter Grenzzone. (C) Chronische autoimmune Hepatitis mit schwergradiger entzündlicher Aktivität (Grad 4 nach Desmet); Destruktion der Grenzzone, lymphoplasmazelluläres Entzündungszellinfiltrat und Ausbildung pseudoazinärer Hepatozytenstrukturen (Pfeil). (D) Chronische Hepatitis infolge Medikamenteneinnahme (hier Langzeit-Nitrofurantointherapie) mit mäßiger entzündlicher Aktivität; Destruktion der Grenzzone und mäßiggradiges entzündungszelliges Infiltrat mit Eosinophilennachweis (Pfeile).

\section{Erläuterung (s. Tab. 3; Abb. 2)}

Die entzündliche Aktivität chronischer Hepatitiden ist in den meisten Fällen variabel über die Zeit und kann sowohl ab- als auch zunehmen. Die Biopsie zeigt somit eine Momentaufnahme, die aber die entzündliche Potenz der bestehenden Hepatitis wiedergibt. Als isolierter Wert ist das Grading deshalb von geringerer Bedeutung als das Fibrosestadium, erlaubt aber zusammen mit diesem auch dann prognostische Aussagen, wenn Infektionszeitpunkt oder Erstmanifestation einer chronischen Hepatitis nicht bekannt sind.

Als technische Voraussetzung für das histologische Grading ist die H.-E.-Färbung eines genügend großen Biopsiezylinders ausreichend. Eine zusätzliche PAS-Reaktion nach Diastase-Verdau zur Identifikation zeroidspeichernder Makrophagen ist hilfreich.

Unterschiedliche deskriptive und numerische Gradingscores kommen zur Anwendung. Die am häufigsten verwendeten Gradingsysteme sind der modifizierte Hepatitis-Aktivitäts-Index
(mHAI) [21], der in Analogie hierzu verwendbare, deskriptive Desmet/Scheuer-Score [19], der sog. METAVIR-Score [22] und der Score nach Batts und Ludwig [20]. Für die Routinediagnostik hat sich der deskriptive Desmet/Scheuer-Score bewährt (minimal, mild, mäßig, schwer; Grad 1-4). Er ist gut reproduzierbar [24] und lässt sich mit den für alle chronischen Hepatitiden definierten Entzündungskriterien des mHAI korrelieren [19]. Im deutschsprachigen Raum besitzt er die höchste Akzeptanz. Die alleinige Verwendung des mHAI, der eine Weiterentwicklung des sog. Knodell-Scores [25] darstellt, ist bei einer Spanne von 0-18 Scorepunkten in der Routinediagnostik weniger praktikabel. Der ebenfalls gut reproduzierbare METAVIR-Score ist nur für die chronische Hepatitis C evaluiert [22] und in den Einzelkriterien nicht exakt definiert bzw. validiert. Der Score nach Batts und Ludwig ist im deutschsprachigen Raum wenig gebräuchlich. In beiden letztgenannten Scores wird die Dichte des portalen Entzündungsinfiltrats nicht berücksichtigt, obwohl es ein Hauptkriterium der Chronizität ist und seine Bedeutung für den Verlauf chronischer Hepatitiden nicht geklärt ist. Eine fehlende 

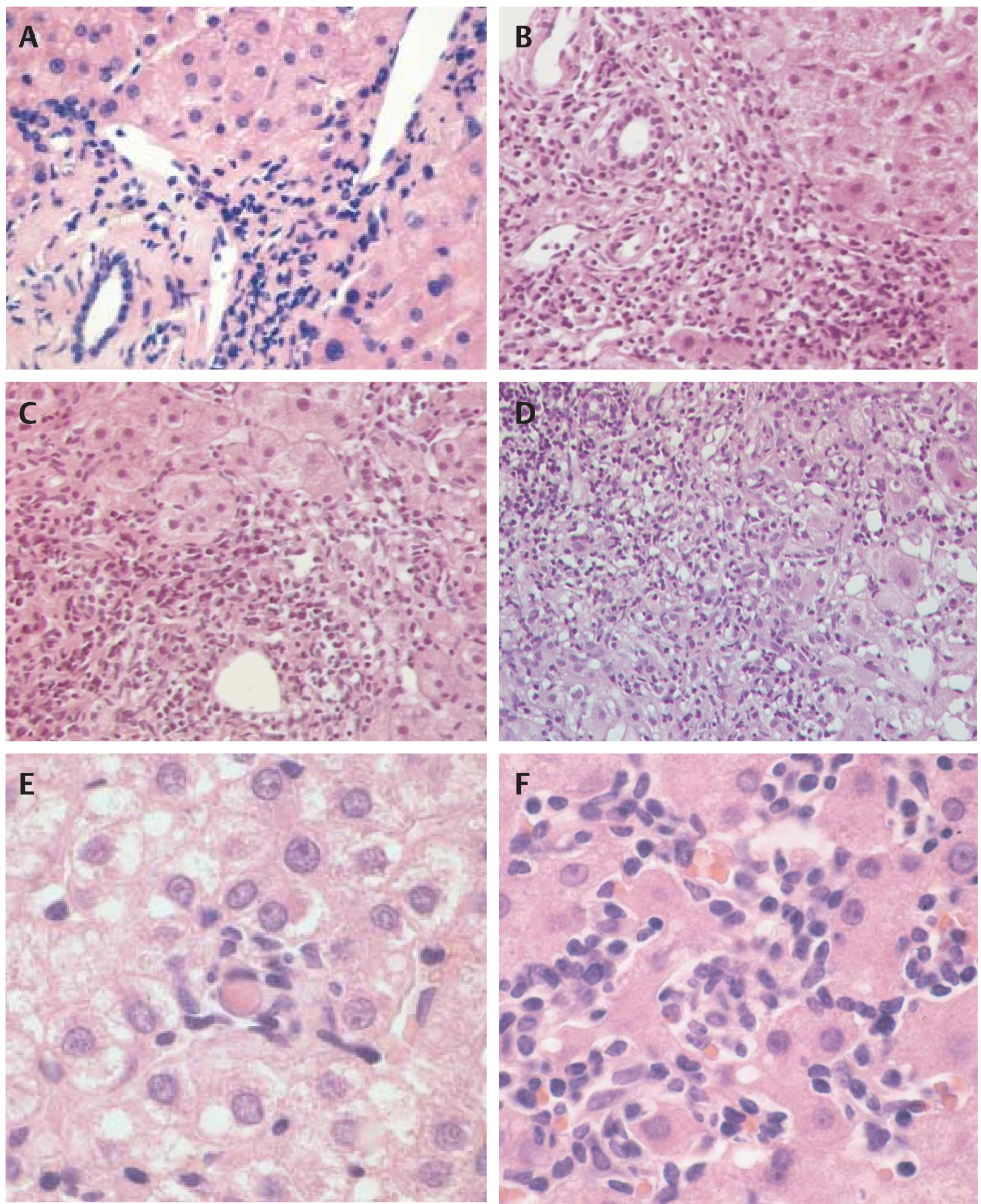

Abb. 2 Entzündliche Aktivität (Grading) der chronischen Hepatitis (s. auch Tab. 3); (A-D) portale und Grenzzonenaktivität, (E), (F) intraazinäre Aktivität; HE-Färbung).

(A) Diskrete portale Lymphozyteninfiltration und intakte Grenzzone bei Hepatitis C mit minimaler entzündlicher Aktivität (Grad 1 nach Desmet). (B) Mäßige portale Lymphozyteninfiltration und fokale Grenzzonenaktivität bei chronischer Hepatitis C mit geringer entzündlicher Aktivität (Grad 2 nach Desmet). (C) mäßige portale Lymphozyteninfiltration mit kontinuierlicher Grenzzonenaktivität bei chronischer Hepatitis C mit mäßiger entzündlicher Aktivität (Grad 3 nach Desmet). (D) Schwergradige entzündungszellige Infiltration und komplette Grenzzonendestruktion bei chronischer autoimmuner Hepatitis mit schwergradiger entzündlicher Aktivität (Grad 4 nach Desmet). Intraazinäre entzündliche Aktivität mit Einzelzelluntergang (Apoptose) (E) und einer Gruppennekrose mit mehr als 10 beteiligten Hepatozyten (F) bei chronischer Hepatitis C. 


\begin{tabular}{|c|c|c|c|c|}
\hline \multicolumn{5}{|c|}{ A. Grading (nach Desmet et al. [19]) } \\
\hline Grad & verbal & $\begin{array}{l}\text { analoger } \\
\text { HAI-Score }\end{array}$ & \multicolumn{2}{|l|}{ histologische Merkmale* } \\
\hline 1 & minimal & $1-3$ & \multicolumn{2}{|c|}{$\begin{array}{l}\text { geringe portale Entzündungszellinfiltration, keine oder minimale azinäre Parenchym- } \\
\text { zelluntergänge oder Entzündungszellinfiltrate, keine Grenzzonenhepatitis }\end{array}$} \\
\hline 2 & $\begin{array}{l}\text { mild/ } \\
\text { geringgradig }\end{array}$ & $4-8$ & \multicolumn{2}{|c|}{$\begin{array}{l}\text { geringe oder mäßige portale Entzündungszellinfiltration, geringe, fokale Grenz- } \\
\text { zonenhepatitis, einzelne parenchymatöse Einzelzellnekrosen, keine Gruppennekrosen }\end{array}$} \\
\hline 3 & $\begin{array}{l}\text { mäßig/ } \\
\text { mittelgradig }\end{array}$ & $9-12$ & \multicolumn{2}{|c|}{$\begin{array}{l}\text { erhebliche (mäßige bis schwere) portale Entzündungszellinfiltration, erhebliche } \\
\text { Grenzzonenhepatitis, zahlreiche azinäre Einzelzellnekrosen, evtl. einzelne Gruppen- } \\
\text { nekrosen, keine Brücken- oder panlobulären Nekrosen }\end{array}$} \\
\hline 4 & $\begin{array}{l}\text { schwer/ } \\
\text { hochgradig }\end{array}$ & $13-18$ & \multicolumn{2}{|c|}{$\begin{array}{l}\text { schwere portale Entzündungszellinfiltration und Grenzzonenhepatitis, schwere azinä- } \\
\text { re Entzündung mit Gruppennekrosen und evtl. Brücken- und panlobulären Nekrosen }\end{array}$} \\
\hline \multicolumn{5}{|c|}{ B. modifizierter histologischer Aktivitätsindex (nach Ishak et al. [21]) } \\
\hline Gruppe & Kriterien & & & Score \\
\hline \multirow[t]{6}{*}{ A } & \multicolumn{4}{|c|}{ periportale oder periseptale Grenzzonenhepatitis (sog. Mottenfraßnekrosen) } \\
\hline & \multicolumn{3}{|l|}{ keine } & 0 \\
\hline & \multicolumn{3}{|c|}{ fokal, wenige Portalfelder } & 1 \\
\hline & \multicolumn{3}{|c|}{ fokal, Mehrzahl der Portalfelder } & 2 \\
\hline & \multicolumn{3}{|c|}{ kontinierlich um weniger als $50 \%$ der Portalfelder } & 3 \\
\hline & \multicolumn{3}{|c|}{ kontinuierlich um über $50 \%$ der Portalfelder } & 4 \\
\hline \multirow[t]{8}{*}{ B } & \multicolumn{4}{|c|}{ konfluente Nekrosen } \\
\hline & \multicolumn{3}{|l|}{ keine } & 0 \\
\hline & \multicolumn{3}{|l|}{ fokal } & 1 \\
\hline & \multicolumn{3}{|c|}{ Zone-3-Nekrosen, wenige } & 2 \\
\hline & \multicolumn{3}{|c|}{ Zone-3-Nekrosen, zahlreiche } & 3 \\
\hline & \multicolumn{3}{|c|}{ Zone-3- und einzelne portozentrale Brückennekrosen } & 4 \\
\hline & \multicolumn{3}{|c|}{ Zone-3- und multiple portozentrale Brückennekrosen } & 5 \\
\hline & \multicolumn{3}{|c|}{ panazinäre oder multiazinäre Nekrosen } & 6 \\
\hline \multirow[t]{6}{*}{ C } & \multicolumn{4}{|c|}{ fokale kleinherdige lytische Nekrosen, Apoptosen und fokale Entzündungsherde** } \\
\hline & \multicolumn{3}{|l|}{ keine } & 0 \\
\hline & \multicolumn{3}{|c|}{ bis 1 Herd pro Gesichtsfeld ( $10 \times$ Objektiv) } & 1 \\
\hline & \multicolumn{3}{|c|}{2 bis 4 Herde pro Gesichtsfeld ( $10 \times$ Objektiv) } & 2 \\
\hline & \multicolumn{3}{|c|}{5 bis 10 Herde pro Gesichtsfeld $(10 \times)$} & 3 \\
\hline & mehr als $10 \mathrm{H}$ & de pro Gesic & tsfeld $(10 \times)$ & 4 \\
\hline $\mathrm{D}$ & portale Entzü & & & \\
\hline & keine & & & 0 \\
\hline & gering, wenig & der alle Por & Ifelder & 1 \\
\hline & mäßig, wenig & der alle Por & Ifelder & 2 \\
\hline & mäßig/ausge & gt, alle Port & felder & 3 \\
\hline & ausgeprägt, a & Portalfelder & & 4 \\
\hline maxima & er Score** & & & 18 \\
\hline
\end{tabular}

* angenähert, da mehrere Komponenten (s. HAI-Score) separat gewertet werden

** diffuse lymphozytäre Infiltrate in den Sinusoiden gehen nicht in den Score ein.

entzündliche Aktivität (entsprechend einem Grad 0) kann nach Desmet/Scheuer-Score nur bei Immunsuppression beobachtet werden. Allerdings ist dann die Diagnose einer chronischen Hepatitis nach pathomorphologischen Kriterien nicht möglich. Der METAVIR-Score wie auch der Score nach Batts und Ludwig definieren dagegen eine fehlende entzündliche Aktivität (A0 bzw. Grad 0), wobei keine Leberzelluntergänge, aber durchaus eine portale Rundzellinfiltration vorliegen dürfen. Dies suggeriert eine Graduierung der Chronizität und ein neues Analogon zur früheren chronisch-persistierenden Hepatitis (s.u.), wofür es aber keine rationale Grundlage gibt. Unter Abwägung aller Vorund Nachteile empfiehlt die Konsensuskommission die Verwendung des Desmet/Scheuer-Scores in der diagnostischen Praxis. Der Score sollte verbal (minimal, mild, mäßig, schwer) und numerisch (1-4) ausgedrückt werden. Das Ausmaß der entzündlichen Aktivität repräsentiert ein Kontinuum und kann über die Zeit Veränderungen unterworfen sein. Daher ist die früher übli- che Unterscheidung [23] in eine chronisch-persistierende und eine chronisch-aktive/aggressive Hepatitis obsolet [26].

Bei der Hepatitis B kann eine stärkere entzündliche Aktivität mit besonderen serologischen und viralen Parametern korrelieren (z.B. bei sog. HBe-Minusmutanten mit HBe/anti-e-Konversion oder Deltavirus-Koinfektion).

\section{Staging}

\section{Konsensus}

Das Staging beschreibt das Ausmaß der Fibrose bzw. der Architekturstörung der chronisch entzündeten Leber. Dieses sollte in einem diagnostischen Score festgehalten werden. Zur Dokumentation werden die Scoringsysteme nach Desmet et al. [19], Batts und Ludwig [20], Ishak et al. (sog. modifizier- 
ter Fibrosescore) [21] und der sog. METAVIR-Fibrosescore (nur bei Hepatitis C) [27] akzeptiert. Es wird empfohlen, den Score nach Desmet et al. [21] anzuwenden (C).

\section{Erläuterung (s. Tab. 4; Abb. 3)}

Das Stadium der Fibrose stellt unter Berücksichtigung des Patientenalters, besser auch des Grades der nekroinflammatorischen Aktivität und idealerweise bei bekanntem Erkrankungsbeginn oder bei Vorliegen einer Verlaufsbiopsie nach 3-5 Jahren das wichtigste histopathologische Kriterium für die Prog- noseabschätzung und somit auch für Therapieindikation und Therapieauswahl dar [6].

Voraussetzung ist eine Bindegewebsfärbung (z.B. van Gieson, Goldner, Sirius-Rot) an einer ausreichend dimensionierten Leberbiopsie. Eine zusätzliche Versilberung (z.B. nach Gomori) ist zur Identifizierung einer gestörten Feinarchitektur (z.B. Regeneratknoten) zu empfehlen. Im Falle einer deutlich ungleichmäßigen, unterhalb der Leberkapsel stärker ausgebildeten Fibrose

\begin{tabular}{|c|c|c|}
\hline Score & verbal & histologische Merkmale \\
\hline 0 & keine Fibrose & keine Faservermehrung \\
\hline 1 & milde/geringgradige Fibrose & portale Faservermehrung, keine Septen \\
\hline 2 & mäßige/mittelgradige Fibrose & inkomplette oder komplette portoportale Fasersepten*, erhaltene Architektur \\
\hline 3 & schwere/hochgradige Fibrose & $\begin{array}{l}\text { septenbildende Faservermehrung mit Architekturstörung }{ }^{* *} \text {, kein Anhalt für kom- } \\
\text { pletten zirrhotischen Umbau }\end{array}$ \\
\hline 4 & Zirrhose & wahrscheinlicher*** oder definitiver zirrhotischer Umbau \\
\hline
\end{tabular}

Tab. 4 Staging (nach Desmet et al. [19] und Scheuer [26])

* unabhängig von Zahl und Breite der Septen

** z. B. portozentrale Septen, Verschiebung der portalen/azinären Architektur (portal-zentralvenöser Abstand)

*** z. B. ohne definitiven Nachweis vollständig bindegewebig separierter Pseudolobuli, aber aufgrund indirekter Zeichen (z. B. fragmentierte, „herausgebrochene“ Pseudolobuli) anzunehmen
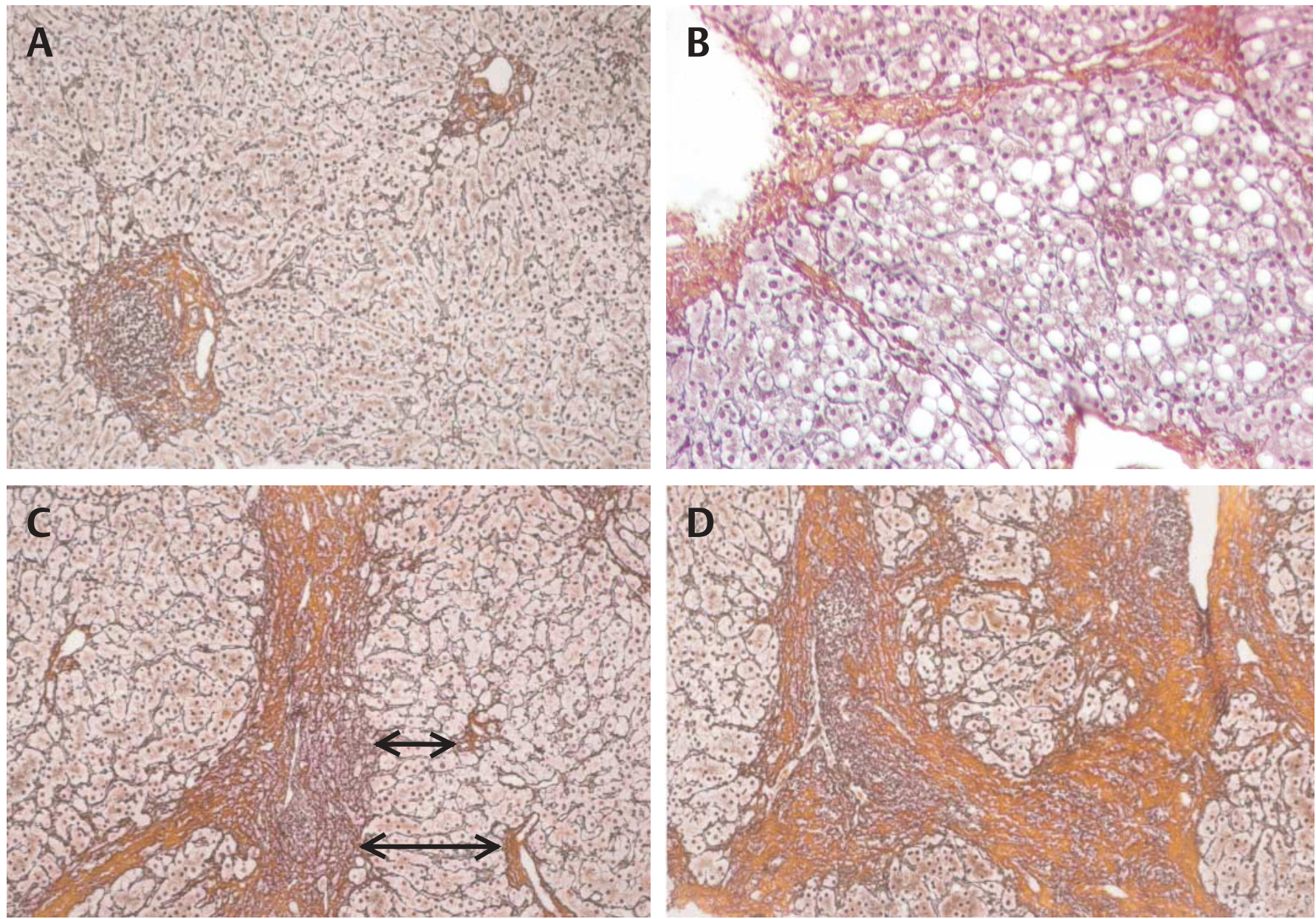

Abb. 3 Fibrose (Staging) am Beispiel der chronischen Hepatitis C (s. auch Tab. 4); modifizierte Gomori-Färbung.

(A) Geringe Fibrose mit ausschließlich portaler Faservermehrung (Stadium 1 nach Desmet). (B) Mäßige Fibrose mit portaler Faservermehrung und zarten, z. T. inkompletten Septen (Stadium 2 nach Desmet). (C) Schwergradige Fibrose mit portaler Faservermehrung, kompletten Septen und Architekturstörung, erkennbar an der teilweise deutlich reduzierten portozentralen Distanz (Doppelpfeile; Stadium 3 nach Desmet). (D) Komplette Zirrhose (Stadium 4 nach Desmet). 
sollten nur tiefere Biopsieanteile ( $>5 \mathrm{~mm}$ Eindringtiefe) zum Staging herangezogen werden.

Das 5-stufige Staging nach Desmet et al. [19] (abgeleitet n. Scheuer [26]) hat sich bewährt, ist einfach durchzuführen und gut reproduzierbar [24]: keine Fibrose (Grad 0), nur portale Fibrose (mild, Grad 1), septenbildende Fibrose ohne Architekturstörung (mäßig, Grad 2), septale Fibrose mit Architekturstörung (schwer, Grad 3) und Zirrhose (Grad 4). Andere 5-stufige Stagingsysteme (METAVIR-Fibrosescore [27], Score nach Batts und Ludwig [20]) sind weit gehend analog, aber unschärfer definiert. Der 7-stufige modifizierte Fibroseindex [21] ist weniger verbreitet und bietet trotz einer weiteren Scorestufe im Bereich der nichtseptenbildenden Fibrose und der zusätzlichen Definition einer inkompletten Zirrhose keinen diagnostisch relevanten Informationsgewinn gegenüber dem einfacheren 5-stufigen Score. Die Konsensuskommission empfiehlt nach Abwägung der Vor- und Nachteile die Verwendung des Scores nach Desmet et al. [19] für die diagnostische Praxis. Der Score sollte verbal und numerisch ausgedrückt werden.

Das Stadium 4 (Zirrhose) umfasst eine große Spannbreite an Bindegewebsneubildung bzw. Parenchymverlust, die in keinem der o.g. diagnostischen Scores weiter unterteilt wird, obwohl das Ausmaß der Faserablagerung stark variieren kann. Diese sind da- her für Therapiestudien bei Patienten mit fortgeschrittener Fibrose oder Zirrhose unbrauchbar. Zu diesem Zweck kann der semiquantitative Score nach Chevallier et al. [28] eingesetzt werden, der selbst bei manifester Zirrhose eine große diagnostische Spannbreite aufweist (15-35 Scorepunkte) und sinnvolle Unterscheidungen erlaubt. Morphometrische oder digitale Bildanalysen zur Fibrosequantifizierung sind abhängig von der Qualität der Bindegewebsfärbung und aufgrund der im Verlauf oft erheblich schwankenden Lymphozytendichte in Portalfeldern und fibrösen Septen fehleranfällig.

Konkurrierende Erkrankungen

\section{Konsens}

Zusätzliche Erkrankungen der Leber können den Progress einer chronischen Hepatitis beschleunigen und deren Prognose verschlechtern (B). Sämtliche Hinweise auf eine zusätzliche Schädigung müssen beschrieben und gewertet werden (C).

\section{Erläuterung (Abb. 4)}

Histomorphologische Zeichen zusätzlicher Schädigungen sind pathologische Ablagerungen (z.B. Eisen, Kupfer, Amyloid), intrazelluläre Einschlüsse (Fett, alpha1-Antitrypsin) oder atypische Fibrosemuster. Kompatibel mit der Diagnose einer chronischen
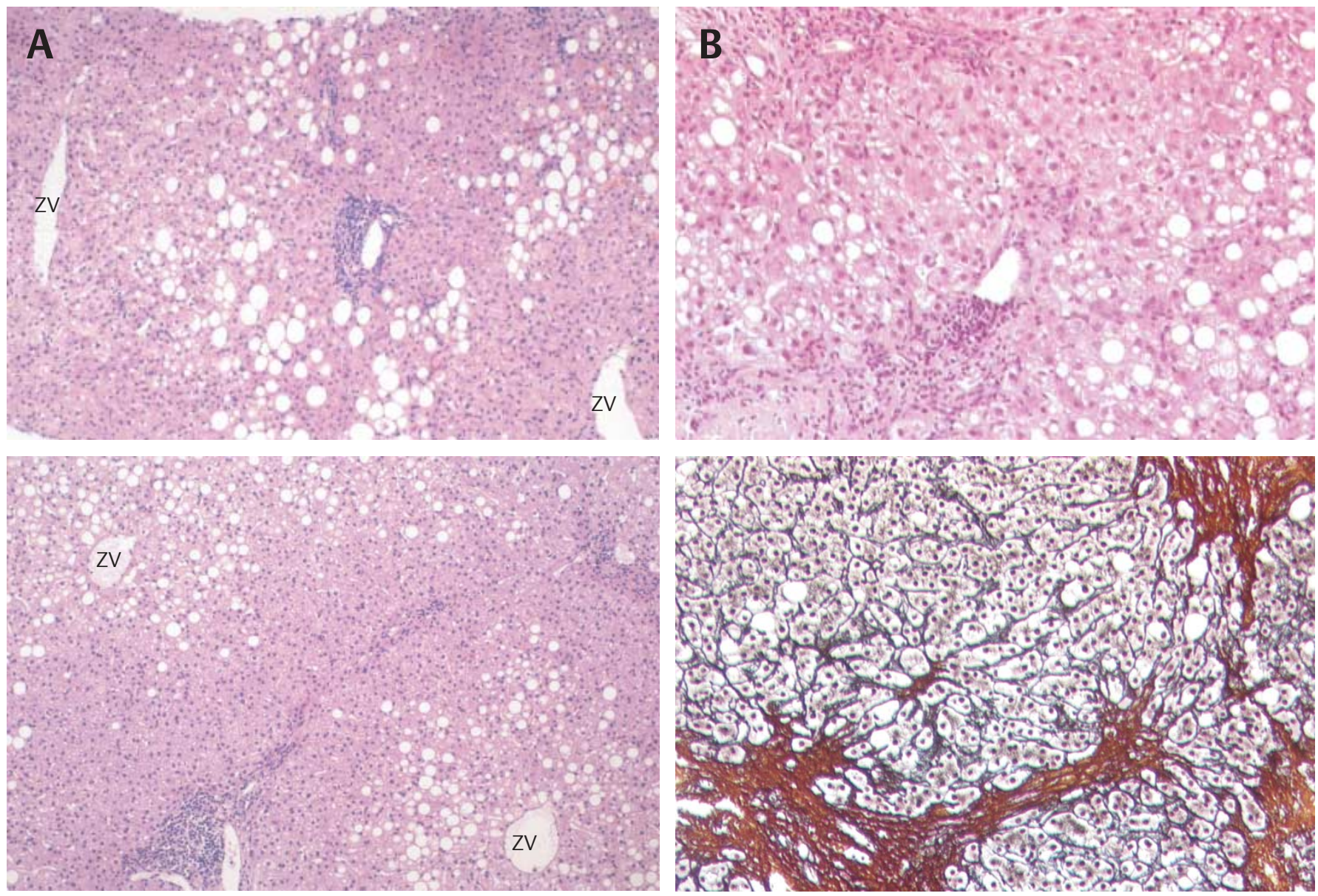

Abb. 4 Histologische Anzeichen der Komorbidität bei chronischer Hepatitis C.

(A) Midzonale und periportale Verfettung vereinbar mit chronischer Hepatitis C (oben) und perivenuläre (Zone 3; ZV = Zentralvenen) Verfettung bei chronischer Hepatitis C und zusätzlichem Alkoholabusus. (B) Prominente Nichtparenchymzellen und perisinusoidale („perizelluläre“) Fibrose infolge zusätzlichen Alkoholabusus bei chronischer Hepatitis C (oben: HE-Färbung, unten: modifizierte Gomori-Färbung). 
Tab. 5 Beispieldiagnosen

Beispiel A:
Diagnose:
chronische Hepatitis (HCV-Infektion nach klin. Angabe) mit milder entzünd-
licher Aktivität (Grad 2 nach Desmet) und milder/portaler Fibrose (Stadium 1
nach Desmet).
Kritischer Bericht/epikritische Bemerkung:
kein Nachweis eines zirrhotischen Umbaus. Kein Anhalt für Malignität.
Beispiel B:
Diagnose:
chronische Hepatitis B mit mäßiger entzündlicher Aktivität (Grad 3 nach
Desmet) und schwerer Fibrose/portaler und septenbildender Fibrose mit
Architekturstörung (Stadium 3 nach Desmet) sowie zusätzlicher mäßiger
Parenchymverfettung und perisinusoidaler Fibrose.
Kritischer Bericht/epikritische Bemerkung:
Parenchymverfettung und perisinusoidale Fibrose sind von der chronischen
Hepatitis B unabhängig; am ehesten sind sie Folge einer chronischen alko-
holtoxischen Schädigung. Am vorliegenden Biopsiegewebe kein Nachweis
eines kompletten zirrhotischen Umbaus. Kein Anhalt für Malignität.
Beispiel C:
Diagnose:
chronische Hepatitis mit schwerer entzündlicher Aktivität (Grad 4 nach
Desmet) und milder/portaler Fibrose (Stadium 1 nach Desmet).
Kritischer Bericht/epikritische Stellungnahme:
Das histologische Bild ist mit einer autoimmunen Hepatitis (klin. Fragestel-
lung bei hochtitrigen Autoantikörpern und Gammaglobulinerhöhung) gut
vereinbar. Im Rahmen des Scoringsystems für die autoimmune Hepatitis
(International Autoimmune Hepatitis Group 1999 ) ist die Histologie mit + 3
zu bewerten. Kein Nachweis eines zirrhotischen Umbaus. Kein Anhalt für
Malignität.

Hepatitis sind geringe Eisenablagerungen in Endothelien (bei chronischer HCV-Infektion), Milchglashepatozyten und sog. „sanded nuclei“ (bei chronischer HBV-Infektion) und Parenchymverfettung (bei HCV-Infektion). Es gibt Hinweise darauf, dass die HCV-assoziierte Verfettung periportal akzentuiert oder diffus verteilt ist. Eine vorwiegend perivenuläre (zentrolobuläre) Verfettung ist verdächtig auf das Vorliegen einer alkoholischen oder nichtalkoholischen (nichthepatitischen) Genese (z. B. Fettstoffwechselstörung, Diabetes mellitus, Medikamente) und sollte eine diesbezügliche Stellungnahme induzieren. Eine weit gehend entzündungsunabhängige, perisinusoidale (Maschendraht-)Fibrose ist ebenfalls verdächtig auf eine alkoholische oder (seltener) nicht-alkoholische Genese, auch in Abwesenheit einer signifikanten Verfettung.

\section{Besonderheiten}

\section{Konsens}

In der Regel sind für die morphologische Diagnostik einer chronischen Hepatitis die unter Entnahme und Bearbeitung des Biopsiegewebes beschriebenen konventionellen Verfahren ausreichend. In besonderen Fällen können in Absprache mit dem behandelnden Arzt zusätzliche Spezialuntersuchungen sinnvoll sein und meist auch am formalinfixierten Biopsiegewebe durchgeführt werden (C).

\section{Erläuterung}

Diese Untersuchungen können z.B. umfassen:

- Nachweis viraler Nukleinsäuren (HCV [inkl. Subtypisierung], seltener EBV oder HBV [in unklaren Fällen]);
- Nachweis von Mutationen (falls kein Vollblut direkt zur Verfügung steht; z.B. bei Verdacht auf genetische Hämochromatose);

- Klonalitätsanalysen bei Verdacht auf Leberinfiltration durch ein malignes Lymphom.

Diese Untersuchungen erfordern spezifische Voraussetzungen in Bezug auf molekularpathologische Expertise, Assaydesign, Kontrollen und Kontaminationsvermeidung [29] und sind daher entsprechend eingerichteten Zentren vorbehalten.

\section{Abschließende Bemerkung}

Eine Aktualisierung der vorliegenden Leitlinie ist nach einem Zeitraum von drei Jahren nach Erscheinen vorgesehen.

\section{Mitglieder der Expertenkommission (alphabetisch)}

\section{Leitung}

1. Prof. Dr. H. P. Dienes (Institut für Pathologie, Universität zu Köln; Fachvertreter Pathologie HepNet)

2. Prof. Dr. W. E. Fleig (Klinik und Poliklink für Innere Medizin I, Universität Halle-Wittenberg; Fachvertreter und Konsensusbeauftragter der DGVS)

3. Prof. Dr. P. Schirmacher (Institut für Pathologie, Universität zu Köln, Fachvertreter DGP; Vorstand AG Gastroenterologische Pathologie der DGP)

\section{Weitere Mitglieder der Expertenkommission}

4. Prof. Dr. M. Burdelski (Klinik und Poliklinik für Kinder- und Jugendmedizin, Universitätsklinikum Hamburg-Eppendorf; Vertreter pädiatrische Hepatologie)

5. Prof. Dr. W. H. Caselmann (Medizinische Klinik und Poliklinik I, Allgemeine Innere Medizin, Universität Bonn; Kongresssekretär der DGVS 2002)

6. Prof. Dr. H. Denk (Institut für Pathologische Anatomie, Universität Graz Österreich; Fachvertreter Pathologie Österreich)

7. Dr. A. Donner (Institut für Pathologie, Universität Düsseldorf; Fachvertreter universitärer Pathologen)

8. Dr. V. Dries (Institut für Pathologie, Heidenheimer Str. 59, Ulm; Fachvertreter nichtuniversitärer Pathologen [Niederlassung])

9. PD Dr. G. Faller (Pathologisches Institut, Universität Erlangen-Nürnberg; Vorstand AG Gastroenterologische Pathologie der DGP)

10. Dr. P. Flemming (Institut für Pathologie, Medizinische Hochschule Hannover; Fachvertreter universitärer Pathologen)

11. Prof. Dr. H. E. Gabbert (Institut für Pathologie, Universität Düsseldorf; Konsensusbeauftragter DGP)

12. Prof. Dr. G. Herrmann (Institut für Pathologie, Klinikum Ludwigsburg; Fachvertreter nichtuniversitärer Pathologen [Prosektur])

13. Prof. Dr. U. Hopf (Medizinische Klinik, Schwerpunkt Hepatologie und Gastroenterologie, Universitätsklinikum Charité [Campus Virchow-Klinikum], Berlin; Fachvertreter Innere Medizin) 
14. Prof. Dr. G. Klöppel (Institut für Allgemeine Pathologie und Pathologische Anatomie, Universitätsklinikum SchleswigHolstein; Campus Kiel; Vorstand Deutsche Gesellschaft für Pathologie)

15. Dr. C. Langner (Institut für Pathologische Anatomie, Universität Graz [Österreich]; Fachvertreter Pathologie Österreich)

16. Prof. Dr. H. Lobeck (Institut für Pathologie, Ernst-von-Bergmann Klinik, Potsdam; Fachvertreter nicht-universitärer Pathologen [Prosektur])

17. Fr. Prof. Dr. J. Lüttges (Institut für Allgemeine Pathologie und Pathologische Anatomie, Universitätsklinikum SchleswigHolstein; Campus Kiel; Vorstand AG Gastroenterologische Pathologie der DGP)

18. Prof. Dr. M. Manns (Abt. Gastroenterologie, Hepatologie und Endokrinologie, Medizinische Hochschule Hannover; Sprecher des HepNet, Fachvertreter Innere Medizin)

19. Prof. Dr. M. Roggendorf (Institut für Virologie, Universität Essen; Fachvertreter Virologie, Vorstand des HepNet)

20. Prof. Dr. J. Rüschoff (Institut für Pathologie, Städtische Kliniken Kassel; Vorsitzender AG Gastroenterologische Pathologie der DGP)

21. Prof. Dr. T. Sauerbruch (Medizinische Klinik und Poliklinik I, Allgemeine Innere Medizin, Universität Bonn; Präsident der DGVS 2002)

22. Fr. Prof. Dr. A. Tannapfel (Institut für Pathologie, Universität Leipzig; Vorstand AG Gastroenterologische Pathologie der DGP)

23. Prof. Dr. L. Terracciano (Institut für Pathologie, Universitätsspital, Basel [Schweiz]; Fachvertreter Pathologie Schweiz)

24. Prof. Dr. C. Wittekind (Institut für Pathologie, Universität Leipzig; Konsensusbeauftragter Berufsverband Deutscher Pathologen)

\section{Literatur}

(Evidenzstärke gemäß Ärztliche Zentralstelle Qualitätssicherung in Klammern)

${ }^{1}$ EASL International Consensus Conference on Hepatitis C. Consensus statement. J Hepatol 1999; 30: 956-961 (IV)

${ }^{2}$ Hopf U, Niederau C, Kleber G et al. Behandlung der chronischen Virushepatitis B/D und der akuten und chronischen Virushepatitis C. Konsensus der Deutschen Gesellschaft für Verdauungs- und Stoffwechselkrankheiten. Z Gastroenterol 1997; 35: 971 - 986 (IV)

${ }^{3}$ National Institutes of Health consensus development conference statement. Management of hepatitis C: 2002. Hepatology 2002; 36: S3-S20 (IV)

${ }^{4}$ Alvarez F, Berg PA, Bianchi FB et al. International Autoimmune Hepatitis Group Report: review of criteria for diagnosis of autoimmune hepatitis. J Hepatol 1999; 31: 929-938 (IV)

${ }^{5}$ Dienstag JL. The role of liver biopsy in chronic hepatitis C. Hepatology 2002; 36: S152 - S160 (IV)

${ }^{6}$ Marcellin P, Asselah T, Boyer N. Fibrosis and disease progression in hepatitis C. Hepatology 2002; 36: S47-S56 (IV)
${ }^{7}$ Pradat P, Poynard T, Alberti A et al. Predictive value of ALT levels for histologic findings in chronic Hepatitis C: a European collaborative study. Hepatology 2002; 36: 973 -977 (III)

${ }^{8}$ Gebo KA, Herlong HF, Torbenson MS. Role of liver biopsy in management of chronic hepatitis C: a systematic review. Hepatology 2002; 36: S161 - S172 (III)

${ }^{9}$ Crawford AR, Lin XZ, Crawford JM. The normal adult human liver biopsy: A quantitative reference standard. Hepatology 1998; 28: 323 - 331 (IIb)

${ }^{10}$ Piccinino F, Sagnelli E, Pasquale G et al. Complications following percutaneous liver biopsy. A multicentre retrospective study on 68,276 biopsies. J Hepatol 1986; 2: 165-173 (III)

11 Miniuk GY, Sutherland LR, Wiseman DA et al. Prospective study of the incidence of ultrasound-detected intrahepatic and subcapsular hematomas in patients randomized to 6 or 24 hours of bedrest after percutaneous liver biopsy. Gastroenterology 1987; 92: 290-293 (Ib)

12 Hederström E, Forsberg L, Florén $\mathrm{CH}$ et al. Liver biopsy complications monitored by ultrasound. J Hepatol 1989; 8: 94-98 (IV)

${ }^{13}$ McGill DB, Rakela J, Zinsmeister AR et al. A 21-year experience with major hemorrhage after percutaneous liver biopsy. Gastroenterology 1990; 99: 1396 - 1400 (IIa)

${ }^{14}$ Conn HO. Liver biopsy: increased risks in patients with cancer. Hepatology 1991; 14: $206-209$ (III)

${ }^{15}$ Gordon SC, Veneri RJ, McFadden RF et al. Major hemorrhage after percutaneous liver biopsy in patients with AIDS. Gastroenterology 1991; 100: 1787 (IV)

16 Özdogan M, Özgür O, Boyacioglu S et al. Percutaneous liver biopsy complications in patients with chronic renal failure. Nephron 1996; 74: $442-444$ (III)

${ }^{17}$ Terjung B, Lemnitzer I, Dumoulin FL et al. Bleeding complications after percutaneous liver biopsy: an analysis of risk factors. Digestion 2003; 67: $138-145$ (III)

18 Guidelines on the use of liver biopsy in clinical practice. Gut 1999; 45 (Suppl. IV): 1-11 (IV)

19 Desmet VJ, Gerber M, Hoofnagle JH et al. Classification of chronic hepatitis: diagnosis, grading and staging. Hepatology 1994; 19: $1513-1520$ (IV)

${ }^{20}$ Batts KP, Ludwig J. Chronic hepatitis - an update on terminology and reporting. Am J Surg Pathol 1995; 19: 1409-1417 (IV)

${ }^{21}$ Ishak KG, Baptista A, Bianchi L et al. Histological grading and staging of chronic hepatitis. J Hepatol 1995; 22: 696-699 (IV)

22 Bedossa P, Poynard T for the METAVIR Cooperative Study Group. An algorithm for the grading of activity in chronic hepatitis C. Hepatology 1996; 24: 289-293 (III)

23 De Groote J, Desmet V, Gedigk P et al. A classification of chronic hepatitis. Lancet 1968; ii: 626-628

${ }^{24}$ Goldin RD, Goldin JG, Burt AD et al. Intra-observer and inter-observer variation in the histopathological assessment of chronic viral hepatitis. J Hepatol 1996; 25: 649-654 (III)

${ }^{25}$ Knodell RG, Ishak KG, Black WC et al. Formulation and application of a numerical scoring system for assessing histological activity in asymptomatic chronic active hepatitis. Hepatology 1981; 1: 431-435

${ }^{26}$ Scheuer PJ. Classification of chronic viral hepatitis: a need for reassessment. J Hepatol 1991; 13: 372 - 374 (IV)

27 The METAVIR Cooperative Group. Inter- and intra-observer variation in the assessment of liver biopsy of chronic hepatitis C. Hepatology 1994; 20: 15-20 (III)

${ }^{28}$ Chevallier M, Guerret S, Chossegros P et al. A histological semiquantitative scoring system for evaluation of hepatic fibrosis in needle liver biopsy specimens: comparison with morphometric studies. Hepatology 1994; 20: 349-355 (IIb)

29 Dries V, von Both I, Müller M et al. Detection of hepatitis C virus in paraffin-embedded liver biopsies of patients negative for viral RNA in serum. Hepatology 1999; 29: 223 - 229 (IIb) 